\title{
The Effect of PVP Molecular Weight on Dissolution Behavior and Physicochemical Characterization of Glycyrrhetinic Acid Solid Dispersions
}

\author{
Xiaoyu Sui $\mathbb{D}^{\mathbb{D}}$, Yan Chu $\mathbb{D}$, Jie Zhang $\mathbb{D}$, Honglian Zhang $\mathbb{D}$, Huiyu Wang $\mathbb{D}$, Tingting Liu $\mathbb{D}$, \\ and Cuiyan Han
}

College of Pharmacy, Qiqihar Medical University, 161006 Qiqihar, China

Correspondence should be addressed to Tingting Liu; 1tting@outlook.com and Cuiyan Han; hancy1001@126.com

Received 8 August 2020; Revised 26 September 2020; Accepted 1 October 2020; Published 31 October 2020

Academic Editor: Ning Zhu

Copyright (C) 2020 Xiaoyu Sui et al. This is an open access article distributed under the Creative Commons Attribution License, which permits unrestricted use, distribution, and reproduction in any medium, provided the original work is properly cited.

\begin{abstract}
The effect of polyvinylpyrrolidone (PVP) as glycyrrhetic acid (GA) solid dispersions carrier at different molecular weights on the dissolution behavior and physicochemical properties was investigated. PVP-GA-SDs prepared with all four molecular weight PVPs displayed good enhancement of dissolution rate and equilibrium solubility compared with pure drug and corresponding physical mixtures. The results showed that the enhancement effect of molecular weight on dissolution rate and equilibrium solubility follows PVP K30 > PVP K60 > PVP K17 > PVP K15. In addition, the dissolution rate and solubility of the SDs with a carrier-drug ratio of $8: 1$ were better than the samples of $4: 1$. The DSC and XRD patterns showed that the crystallization of GA in SDs prepared by PVP K30 and PVP K60 was significantly inhibited, and both were transformed to amorphous. Based on FTIR and Raman detection, a hydrogen-bond between PVP and drug molecules is formed. SEM results showed that there were no significant differences in the appearance of SDs prepared with four PVPs, and no crystalline morphology of GA was seen. In conclusion, the findings of this study demonstrated that the dissolution performance of the PVP-GA-SDs prepared by the solvent method is related to the molecular weight of PVP, and the change in the molecular weight of PVP does not cause a monotonic change in dissolution of GA. The samples with PVP K30 as the carrier have the best dissolution performance.
\end{abstract}

\section{Introduction}

Glycyrrhetinic acid (GA) is one of the main active ingredients extracted from the root or rhizome of licorice [1]. It is also a degradation product of glycyrrhetic acid in the body, which is the main active component of licorice [2]. The molecular formula of GA is $\mathrm{C}_{30} \mathrm{H}_{46} \mathrm{O}_{4}$, and the molecular weight is 470.69 . The structural formula is shown in Figure 1. Studies have shown that glycyrrhetic acid has anti-inflammatory, antiviral, antitumor, and other pharmacological effects [3-5]. Currently, it is often used clinically to treat chronic hepatitis and liver cancer [6]. However, due to the strong hydrophobicity of glycyrrhetic acid, its solubility and dissolution rate are poor. This also limits the oral bioavailability of glycyrrhetic acid [7]. In general, the solubility of hydrophobic drug can be improved by adding a surfactant, but a large amount of solubilizer is prone to side effects such as hemolysis. In order to overcome poor solubility of GA, in recent years, some research on new drug delivery systems of GA have been done, including GA liposomes [8], GA nanoemulsions [9], GA microparticles [10], and GA solid dispersion [11].

The solid dispersions (SDs) technique refers to the uniform dispersion system of drugs in the form of molecules, microcrystallines, amorphous, and other states in a solid carrier. Due to the high dispersion of the drug and the high hydrophilicity of the carrier, the wettability of the drug is improved. Therefore, this technique is an effective method for improving the dissolution and oral bioavailability of poorly soluble drugs. Currently, there are some commercial products based on solid dispersions [12], such as vemurafenib (Zelboraf ${ }^{\circledR}$ ), regorafenib (Stivarga ${ }^{\circledR}$ ), and everolimus (Afinitor ${ }^{\circledR}$ ). Solid dispersions are often prepared by four methods, including the comilling method, melting method, 


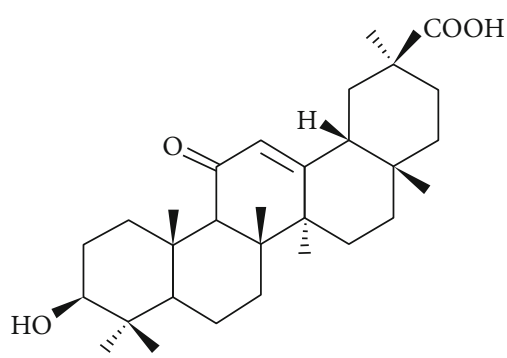

(a)

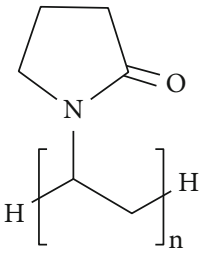

(b)

Figure 1: Chemical structure of glycyrrhetinic acid (a) and polyvinylpyrrolidone (b).

solvent melting method, and solvent method. The solvent method is also called the coprecipitation method, in this method, solid dispersion can be obtained after the evaporation of solvent from the solution containing carrier and drug. Some polymers are hardly used as carriers in the melting method due to their high melting point or thermal decomposition. Because the solvent method can be achieved without using high temperature in the preparation process, this method is suitable for thermally unstable drugs or high melting point carriers $[13,14]$. Currently, the most commonly used carrier in the solvent method is polyvinylpyrrolidone (PVP) [15].

Polyvinylpyrrolidone (PVP) is an amorphous highmolecular polymer, high melting point, stable to heat, easily soluble in water, and many organic solvents. After oral administration, PVP is not absorbed by the gastrointestinal tract and is nontoxic [16]. Commonly used PVP specifications include PVP K15, PVP K17, PVP K30, PVP K60, and PVP K90 (average molecular weight is about 8000 , 10000, 50000, 400000, and 1000000). Until now, studies of these specifications of PVP as solid dispersion carriers have been reported [17-19]. Generally speaking, after the drug is dispersed in PVP, it is easy to form an amorphous solid dispersion due to the strong crystal inhibiting effect of PVP [20]. Studies have shown that the drug release profile of amorphous solid dispersions is driven by the polymer properties. Amorphous solid dispersions show a higher apparent solubility than drug crystalline due to "spring and parachute" effect, which include two key steps, namely, the generation of metastable supersaturation state and inhibition of precipitation [21]. The magnitude of "spring and parachute" effect is highly dependent on the type and molecular weight of the polymer. Hence, it is important to study the influence of molecular weight on the properties of solid dispersions [22].

There are 59 articles found on Scopus using keywords: "PVP" \& "molecular weight" \& "solid dispersion". A total of 14 articles contain research on the relationship between the PVP molecular weight and drug solubility or dissolution, and only 6 studies compared experimentally determined dissolution rates of solid dispersions prepared using more than two molecular weights of PVPs as carriers. Moreover, all researches have focused on synthetic drugs with molecular weights less than 400 and are commonly used in the clinic with antipyretic, anti-inflammatory, and blood pressurelowering effects.
Most of the previous studies [23-26] found that the dissolution rate of drug from the SDs decreases with increasing molecular weight of PVP. A few studies have shown that PVP has a nonmonotonic effect on the dissolution behavior of drugs in solid dispersions [27]. These studies indicate that the effect of PVP molecular weight on the dissolution rate of the drug in the solid dispersions is related to the structural characteristics of the drug.

To date, there are few studies on solid dispersions of glycyrrhetinic acid, and there is no study on the effect of PVP molecular weight on the dissolution behavior of solid dispersions of glycyrrhetic acid or other structurally similar pentacyclic triterpenoids (oleanolic acid, glycyrrhizic acid, ursolic acid, etc.). For this reason, the aim of the present study was to investigate the effect of different molecular weight PVPs as carriers on the dissolution behavior of glycyrrhetic acid solid dispersion by the solvent method. In addition, the differences in physical and chemical properties, especially regarding crystallization inhibition, were further investigated by examining micromorphology and structure properties using SEM, FTIR, XRD, and other identification techniques.

\section{Materials and Methods}

2.1. Materials. GA ( $>98 \%$ pure), Reference compound of GA, PVP K15, PVP K17, PVP K30, and PVP K60 (analytically pure), was obtained from Dalian Meilun Biology technology Co., Ltd (Dalian, China). Ethanol was purchased from Sinopharm Chemical Reagent Co., Ltd. (Beijing, China). Pure water was prepared by a Milli-Q water purification system (Millipore, Bedford, MA, USA).

2.2. Preparation of Solid Dispersions. PVP-GA-SDs were prepared by the solvent method. Briefly, the GA and carrier were weighed based on the formulated amount and placed in an evaporating dish. They were then codissolved in $95 \%(\mathrm{~V} / \mathrm{V})$ ethanol-water solution and heated in a water bath $\left(80 \pm 1^{\circ} \mathrm{C}\right.$ ) with stirring. After removal of ethanol, the mixture was taken out and placed in a vacuum dryer for several days, and then it was milled and sieved with 80 mesh sieves for subsequent experiments.

2.3. Preparation of Physical Mixtures. Physical mixtures (PMs) were prepared by manually mixing of GA and different PVPs which were previously sieved through 80 in a mortar for $5 \mathrm{~min}$, until a homogenous mixture was obtained. 


\subsection{Analysis for Characterization of Solid Dispersions}

2.4.1. Determination of Equilibrium Solubility. Excess sample (pure GA, PMs or SDs, equivalent to $10.0 \mathrm{mg} \mathrm{GA}$ ) was added into $10 \mathrm{~mL}$ of pure water, $\mathrm{PBS}(\mathrm{pH}=6.8)$, and $0.1 \mathrm{~N} \mathrm{HCl}$ solution under magnetic stirring $(300 \mathrm{rpm})$ at $25^{\circ} \mathrm{C}$ in a temperature-controlled water bath until equilibrium was achieved $(48 \mathrm{~h})$. The samples were filtered through a $0.45 \mu \mathrm{m}$ Teflon membrane filter, suitably diluted with methanol, and analyzed via high-performance liquid chromatography (HPLC) as described below. The drug solubility was assayed using a LabAlliance (model Series III) HPLC system (Lab Alliance, Tianjin, China) equipped with a quaternary pump, an autosampler, and a column compartment, coupled to a UV detector. Separation was performed on a C18 column $(4.6 \mathrm{~mm} \times 250 \mathrm{~mm} ; 5 \mu \mathrm{m}$; Dikma Technologies, Beijing, China). The mobile phase comprises methanol, water, and acetic acid $(89: 10: 1, \mathrm{v} / \mathrm{v} / \mathrm{v})$ at a flow rate of $1.0 \mathrm{~mL} \cdot \mathrm{min}^{-1}$. The analytes were detected by a UV detector at $250 \mathrm{~nm}$. A good linearity was found for GA in the range of 0.5 $50 \mu \mathrm{g} \cdot \mathrm{mL}^{-1}$.

2.4.2. In Vitro Dissolution Testing. The dissolution rate was determined using a USP type II Apparatus (RCZ-8A, Tiandatianfa Tech., Ltd., Tianjin, China). $900 \mathrm{~mL}$ of pure water, $0.1 \mathrm{~N}$ $\mathrm{HCl}$ solution, and phosphate buffer solution $(\mathrm{pH}=6.8)$ were used as the dissolution medium $\left(37^{\circ} \mathrm{C} \pm 0.2^{\circ} \mathrm{C}\right)$ for the solid sample (equivalent to $10.0 \mathrm{mg} \mathrm{drug}$ ) with the paddle rotation speed of $100 \mathrm{rpm}$. At predetermined time intervals, $5 \mathrm{~mL}$ aliquots were withdrawn, filtered through a $0.45 \mu \mathrm{m}$ Teflon membrane filter, and analyzed by HPLC. The removed volume was replaced with fresh media to maintain sink conditions. The drug dissolution was performed in triplicate.

2.4.3. Differential Scanning Calorimetry (DSC). DSC measurements were performed on a HSC-1 DSC scanning calorimeter (Hengjiu Instrument, Ltd., Beijing, China). Samples were precisely weighted $(10 \mathrm{mg})$ and were sealed in aluminium crucible. The samples were heated from 25 to $350^{\circ} \mathrm{C}$ at a rate of $10^{\circ} \mathrm{C} \cdot \mathrm{min}^{-1}$ under a nitrogen atmosphere.

2.4.4. X-Ray Diffraction (XRD). The structural properties of samples were obtained using the D8 Focus X-ray diffractometer (Bruker, Germany) with $\mathrm{Cu}-\mathrm{K} \alpha$ radiation. Measurements were performed at a voltage of $40 \mathrm{kV}$ and $40 \mathrm{~mA}$. Samples were scanned from $5^{\circ}$ to $60^{\circ}$, and the scanned rate was $5^{\circ} \cdot \mathrm{min}^{-1}$.

2.4.5. Fourier Transform Infrared Spectroscopy (FTIR). The FTIR spectra of samples were obtained on a Nicolet 6700 FT-IR spectrophotometer (Thermo Scientific, Waltham, MA, USA). Every sample and potassium bromide were mixed by an agate mortar and compressed into a thin disc. The scanning range was $4000-400 \mathrm{~cm}^{-1}$, and the resolution was $4 \mathrm{~cm}^{-1}$.

2.4.6. Raman Spectrometry (RS). FT-Raman spectra were acquired by a Nicolet 6700 FTIR with NXR FT-Raman module $(1064 \mathrm{~nm})$ in the wavenumber range $3700-100 \mathrm{~cm}^{-1}$ with an accumulation of 64 scans with $4 \mathrm{~cm}^{-1}$ spectral resolution.
2.4.7. Scanning Electron Microscopy (SEM). The samples were fixed to an SEM stub with a carbon conductive and sputter-coated with gold using SBC-12 sputter-coater (KYKY Tech., Ltd., Beijing, China). The surface morphology of the sample was then observed by a S-4300 scanning electron microscope (Hitachi, Tokyo, Japan).

\section{Results and Discussion}

3.1. Determination of Equilibrium Solubility. The solubility of a drug in a solid dispersion system is generally greater than that of a pure drug, and the type of dissolving medium also has an influence on the solubility of the drug in a solid dispersion. In this research, the equilibrium solubility of the four kinds of PVP-GA-SDs and the four kinds of PVP-GA-PMs in pure water, PBS, and hydrochloric acid solution were determined as mentioned in Section 2.4.1, respectively. The results are shown in Table 1.

The data in the table showed that the solubility GA in pure water was improved by SDs prepared with PVP at different molecular weights as carrier. Among them, the solubilization effects of PVP K30-GA-SDs and PVP K60-GA-SDs were better than those of PVP K17-GA-SDs and PVP K 15GA-SDs. PVPK30 with a carrier-drug weight ratio of $8: 1$ presented the strongest solubilization ability, and its solubility was $32.06 \pm 3.13 \mu \mathrm{g} \cdot \mathrm{mL}^{-1}$. In a hydrochloric acid solution, PVP K30-GA-SDs and PVP K60-GA-SDs maintained a certain solubilization improvement, but the solubility of other SDs could not be detected, and PVP K30-GA-SDs has significantly higher solubility than PVP K60-GA-SDs. Compared with the above two media, in PBS, SDs had the best solubilization. Similarly, the solubilization effects of PVP K30GA-SDs and PVP K60-GA-SDs are better than those of PVP K15-GA-SDs and PVP K17-GA-SDs. Among all solid dispersion samples, the PVP K30-GA-SDs with a carrierdrug weight ratio of $8: 1$ has the best solubilization effect, with a solubility of $566.51 \pm 34.55 \mu \mathrm{g} \cdot \mathrm{mL}^{-1}$. On the other hand, all physical mixtures are more soluble in PBS than in pure water, and their solubility in hydrochloric acid cannot be detected.

3.2. Determination of In Vitro Dissolution Rate. In general, PBS and hydrochloric acid solution was used as simulated intestinal and gastric fluid, respectively. According to the above results, the equilibrium solubility of each solid dispersion in PBS is higher than in the other two dissolution media. In addition, the use of hydrochloric acid media does not guarantee that all solid dispersions will perform dissolution experiments under sink conditions. In order to satisfy the sink condition, PBS was selected as the dissolution medium in the dissolution determination. The dissolution rates of pure GA, four PMs, and four SDs in PBS are shown in Figure 2.

The experimental results showed that the molecular weight of PVP has a significant effect on the dissolution behavior of PVP-GA-SDs. However, there is no linear relationship between the dissolution rate and molecular weight. The dissolution rate of SDs with carrier-drug ratio of $8: 1$ and $4: 1$ both have the following ordering: dissolution rate 


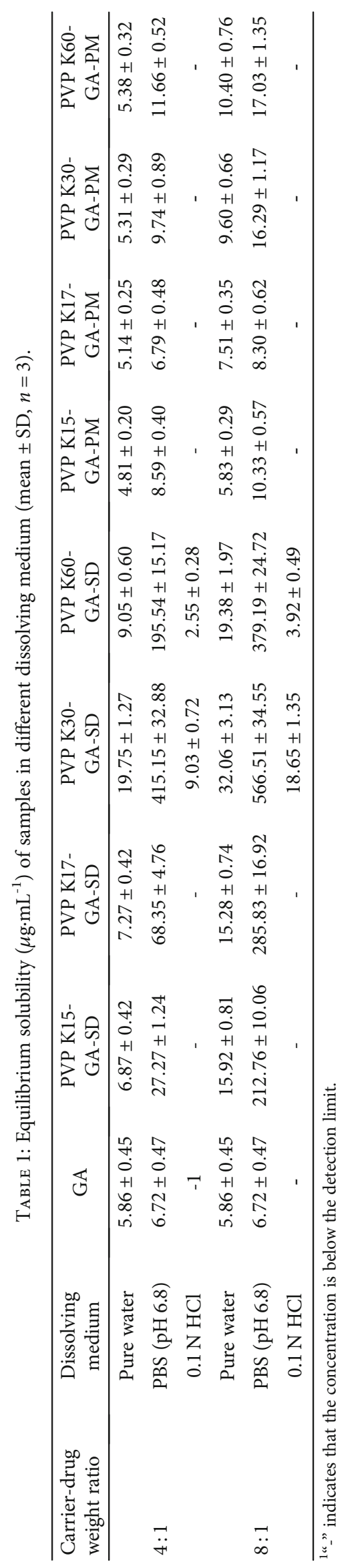




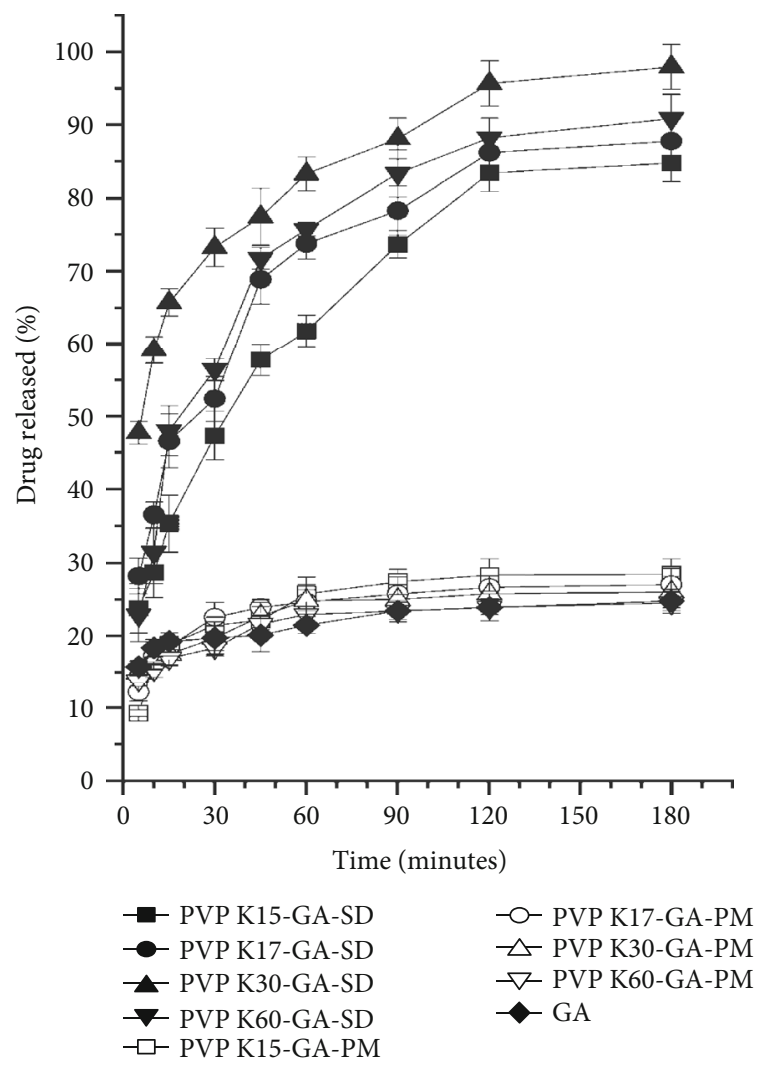

(a)

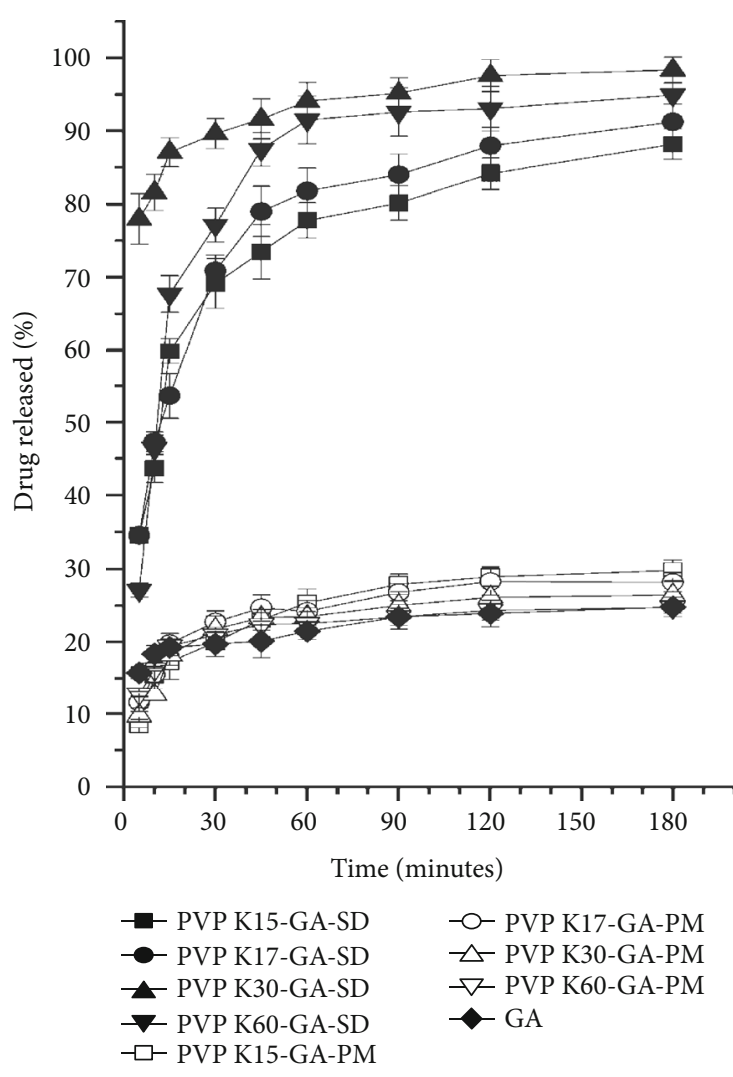

(b)

Figure 2: Dissolution profiles of pure GA, PVP-GA-SDs, and PVP-GA-PMs in phosphate buffer solution. (a) PVP/GA weight ratio of $4: 1$. (b) PVP/GA weight ratio of $8: 1$.

$(\mathrm{K} 30)>$ dissolution rate $(\mathrm{K} 60)>$ dissolution rate $(\mathrm{K} 17)>$ dissolution rate (K15), which is also consistent with the relationship between PVP molecular weight and GA equilibrium solubility in PBS. On the other hand, the carrierdrug weight ratios have an obvious impact on the dissolution rate of SDs. Before $120 \mathrm{~min}$, the dissolution rate of SDs with carrier-drug ratio of $8: 1(W / W)$ was faster than $4: 1(W / W)$ under the same PVP molecular weight condition. When the carrier-drug ratio was $4: 1$, the dissolution rate of SDs with PVP K30 and PVP K60 tended to equilibrium after $120 \mathrm{~min}$. When the drug loading ratio was $8: 1$, the dissolution rate of each SD with PVP K30 and PVP K60 tended to equilibrium after $60 \mathrm{~min}$. Overall, the dissolution rates with PVP K30 as the carrier were the fastest. The cumulative percentage of dissolution of PVP K30-GA-SDs $(8: 1, W / W)$ could reach $81.60 \pm 2.45 \%$ at the 10 th minute.

The pure GA and all PMs were lower than that of SDs, and PMs are a few differences from GA. We observed the maximum percentage of dissolution of PMs prepared with PVP K15 and PVP K17 was slightly higher than samples with the other two PVPs.

3.3. Differential Scanning Calorimetry (DSC). Thermal analysis techniques can be used to speculate change in drug crystallinity or drug-carrier interactions in solid dispersions. Some studies have shown that endothermic melting peaks of drug can be observed in physical mixtures of certain crys- talline drugs and PVP, and once the drug becomes amorphous or drug interacts with the carrier, the corresponding melting point peaks disappear or weaken $[20,28-30]$. Therefore, in this study, we compared the thermal properties of solid dispersions prepared from four different molecular weight PVPs.

In the DSC thermogram (Figure 3), GA showed an absorption peak at $299^{\circ} \mathrm{C}$, which was the melting peak of GA. No obvious characteristic peaks were found for all four PVPs with different molecular weights due to the amorphous nature of PVP. This GA melting peak was also not observed in the physical mixtures of GA with PVP. The same behavior has been reported about the physical mixture of prednisolone with PVP [31, 32]. The reason for this may be low GA content relative to the PVP, leading to a low calorific value, which cannot be displayed in the PM spectra. In addition, SDs showed a similar behavior due to the absence of a GA melting peak, from which it maybe can be inferred that the crystalline structure of the drug in these samples has disappeared. However, this information on whether the crystallinity of GA has changed is not available can only be confirmed with the aid of other techniques such as X-ray diffraction.

3.4. X-Ray Diffraction (XRD) Analysis. Many studies have shown that PVP-drug solid dispersions prepared by solvent methods tend to form a glassy state. Since the carrier of the glassy solid solution has a high viscosity and easily interacts 


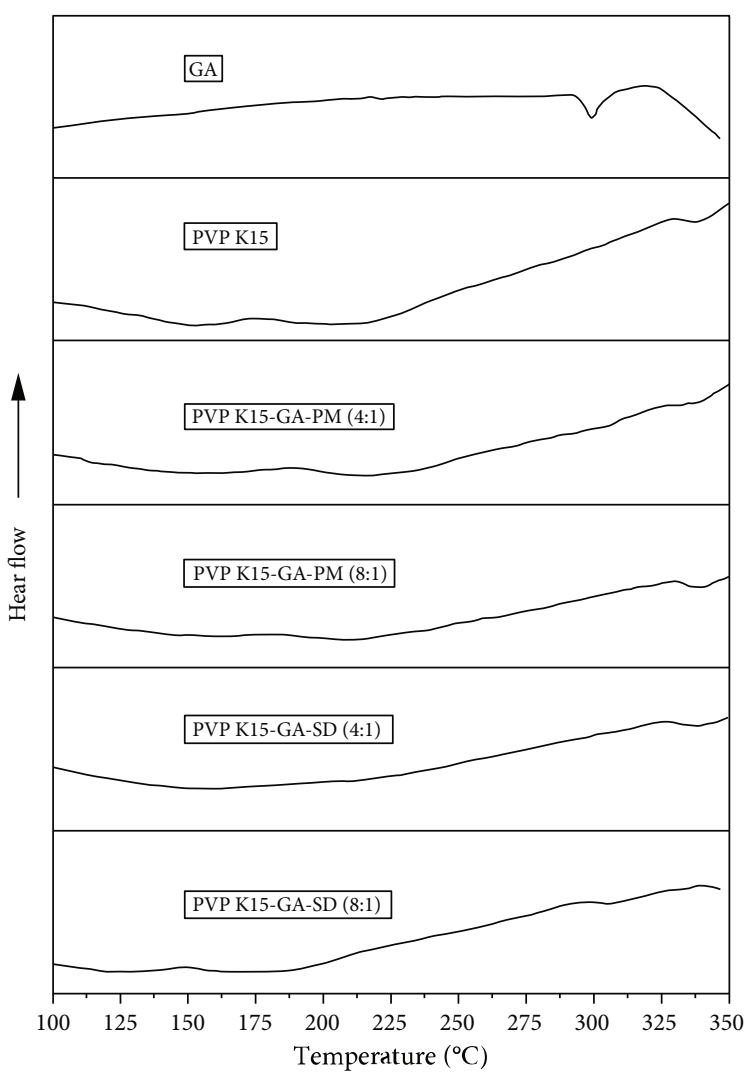

(a)

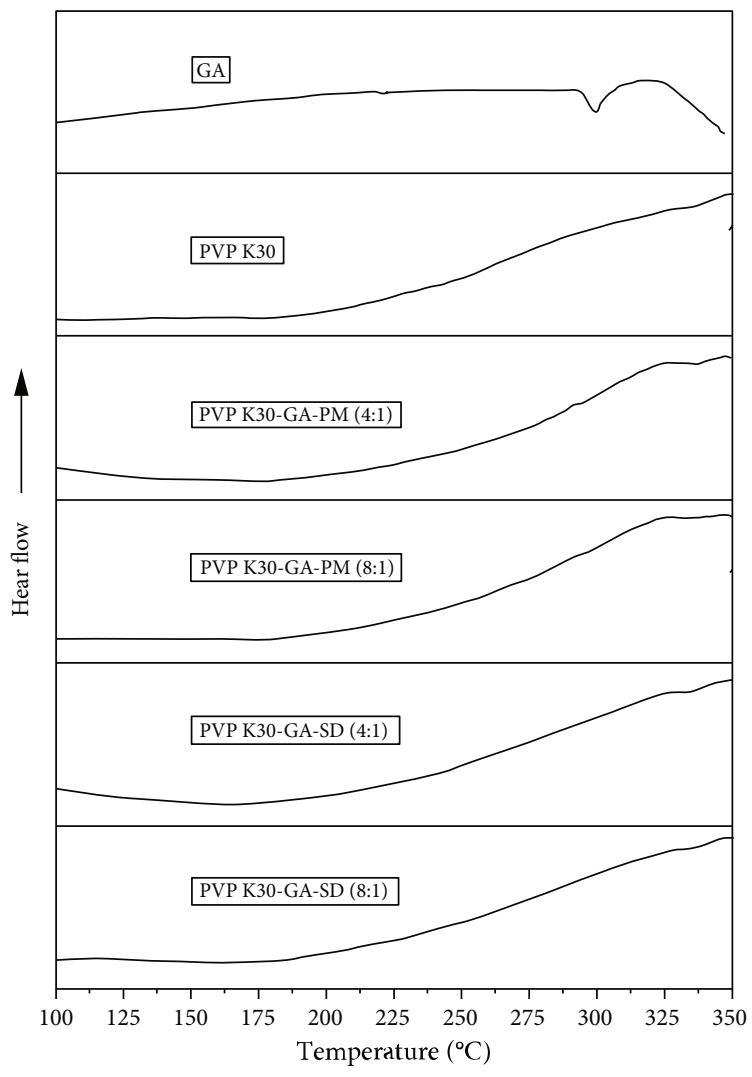

(c)

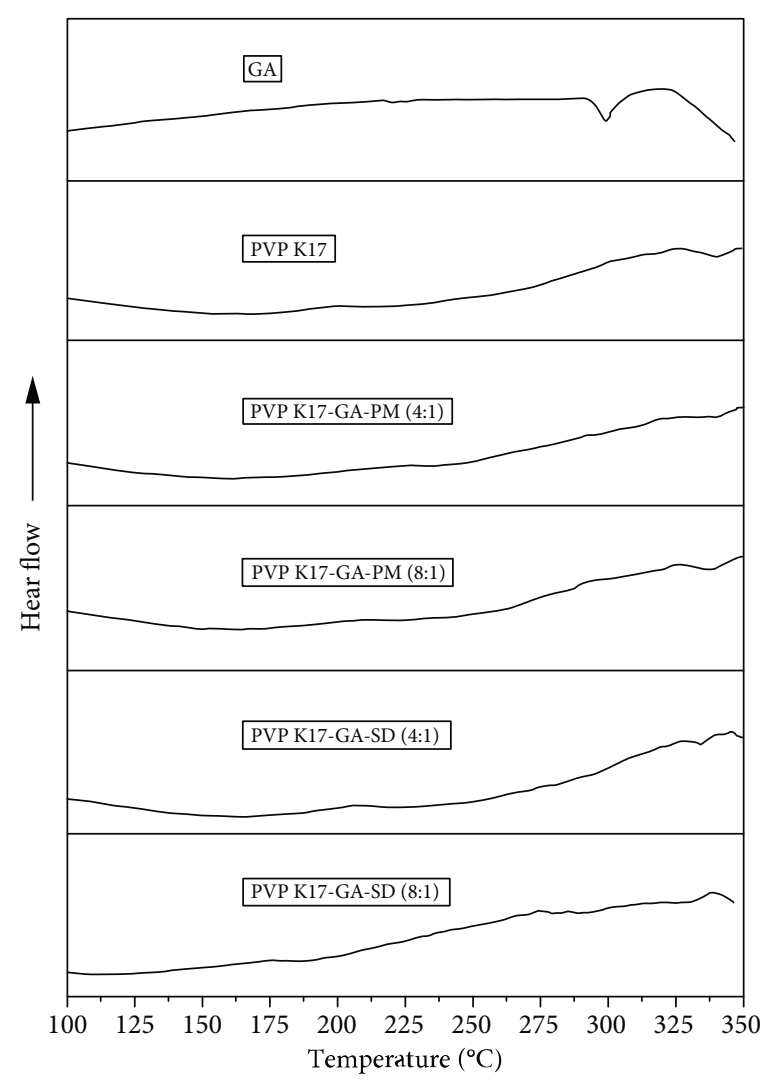

(b)

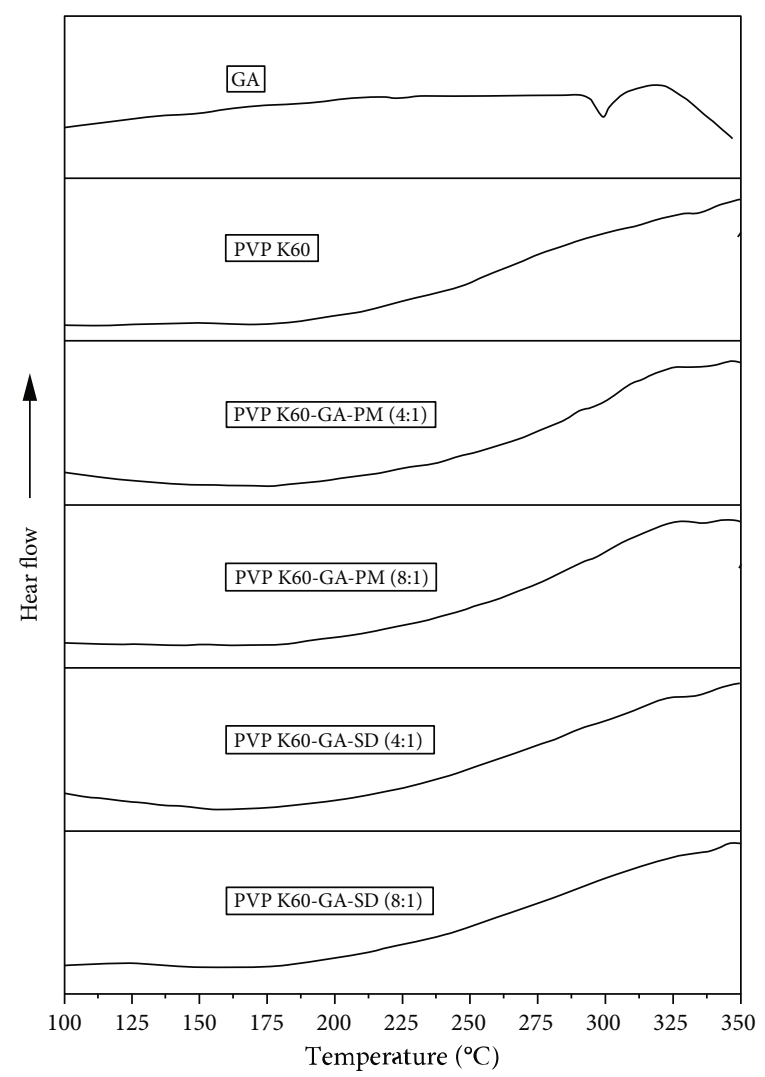

(d)

Figure 3: DSC curves of SDs and PMs of GA prepared using (a) PVP K15, (b) PVP K17, (c) PVP K30, and (d) PVP K60. 


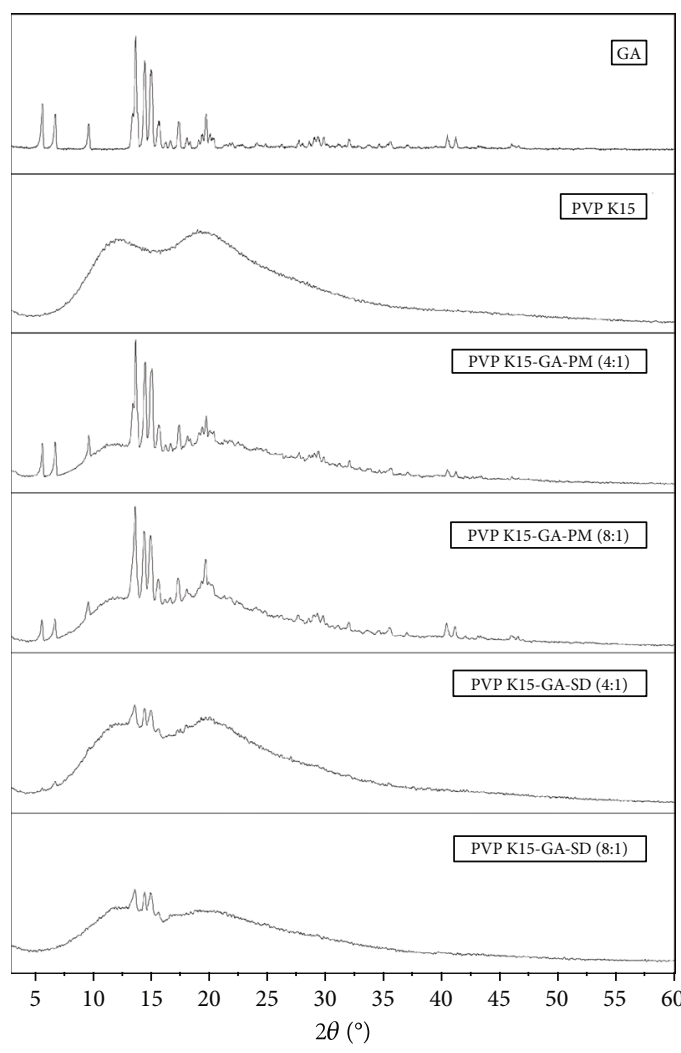

(a)

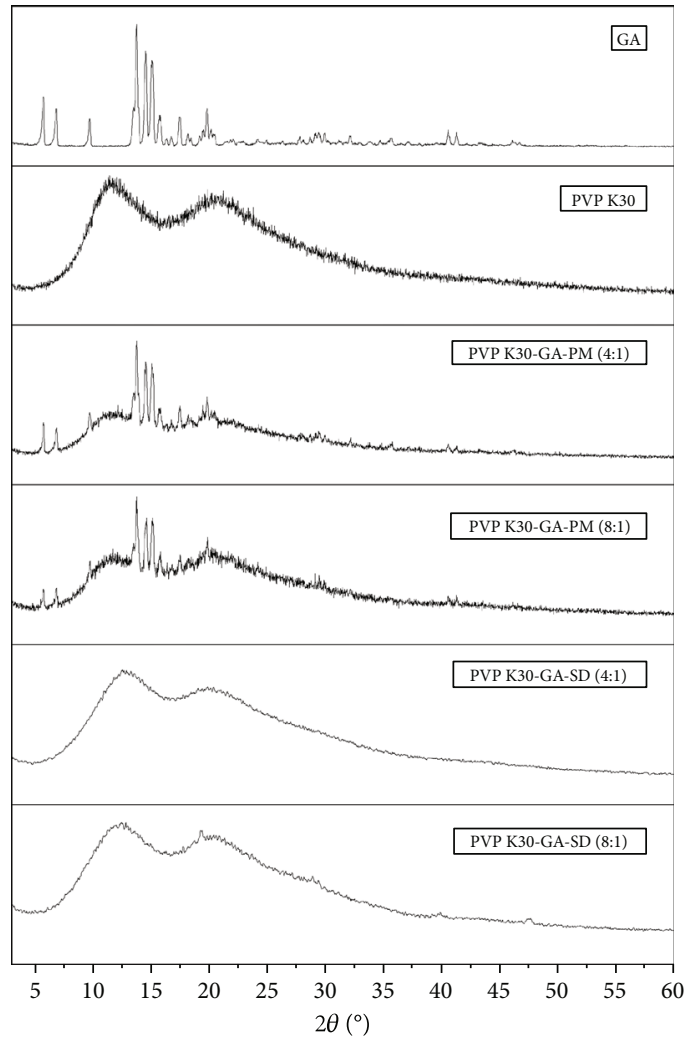

(c)

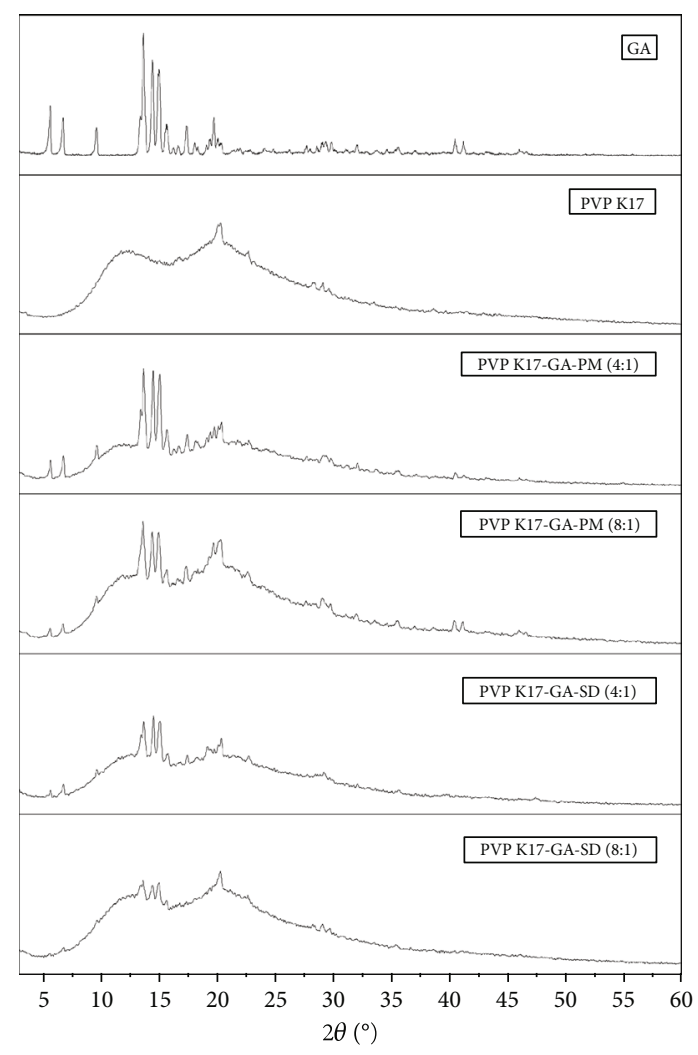

(b)

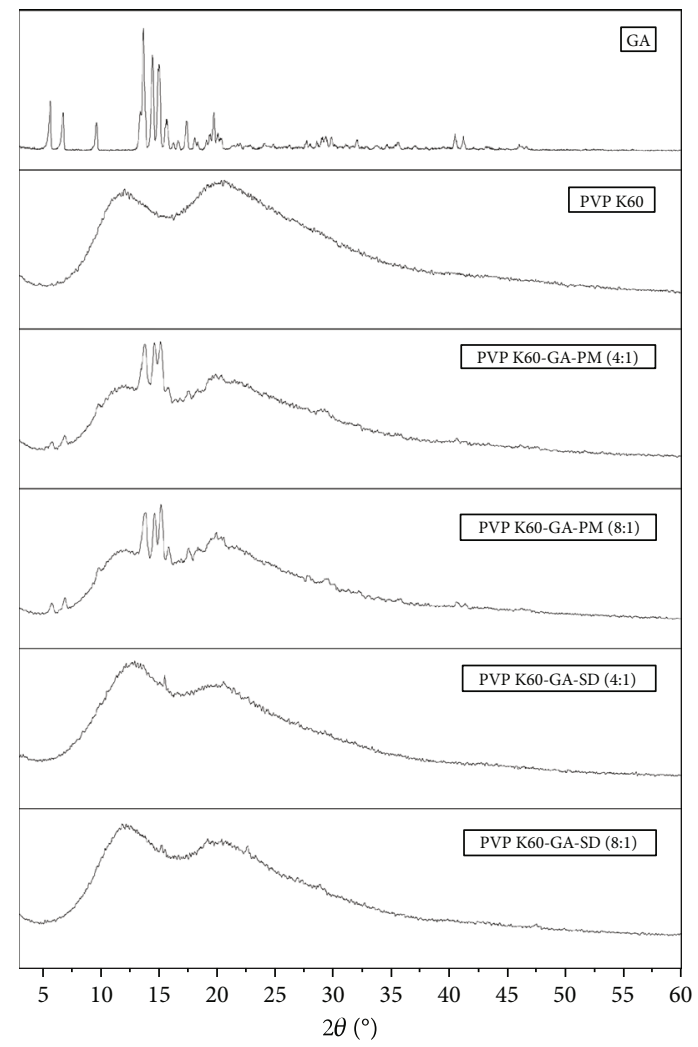

(d)

FIgURe 4: X-ray diffractogram of SDs and PMs of GA prepared using (a) PVP K15, (b) PVP K17, (c) PVP K30, and (d) PVP K60. 


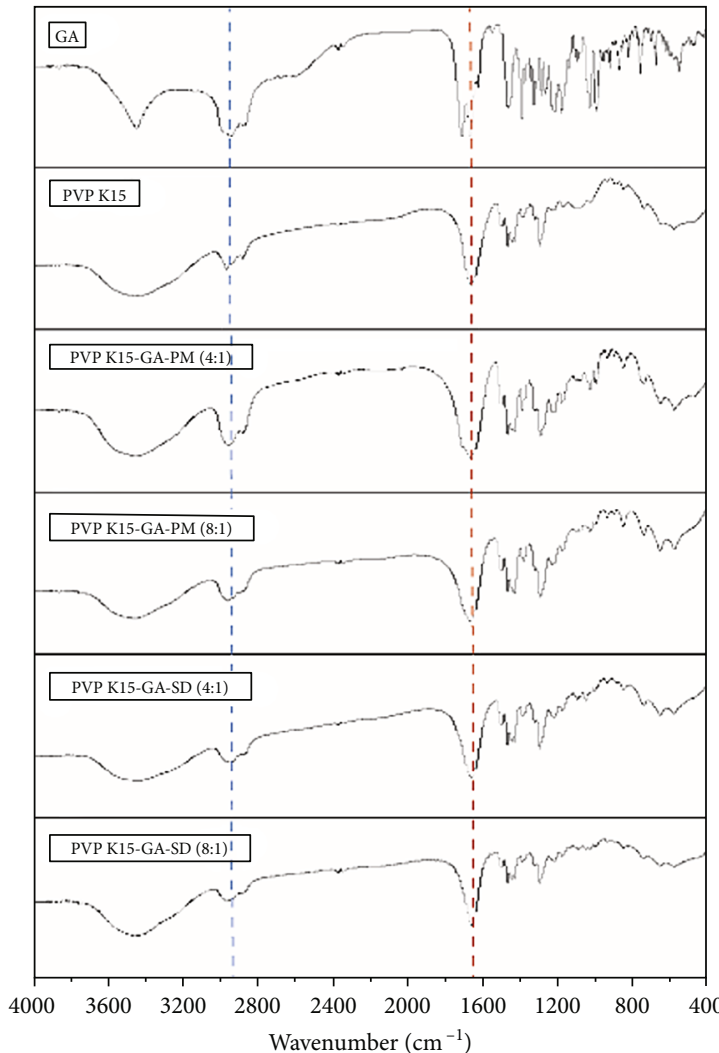

(a)

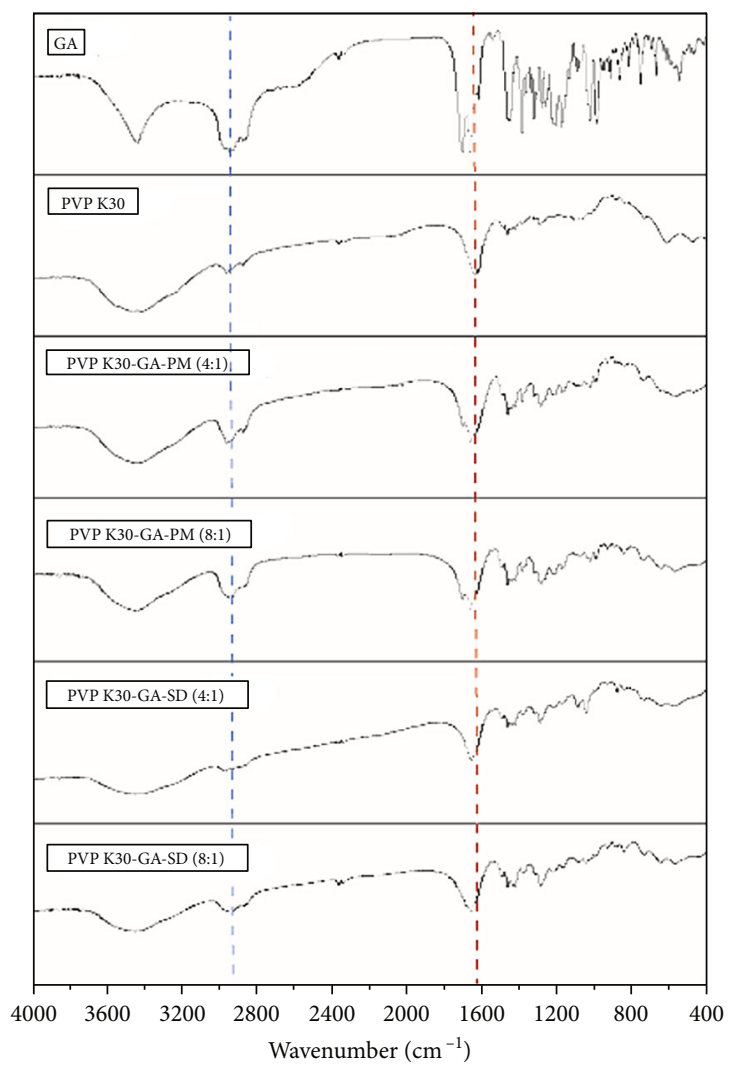

(c)

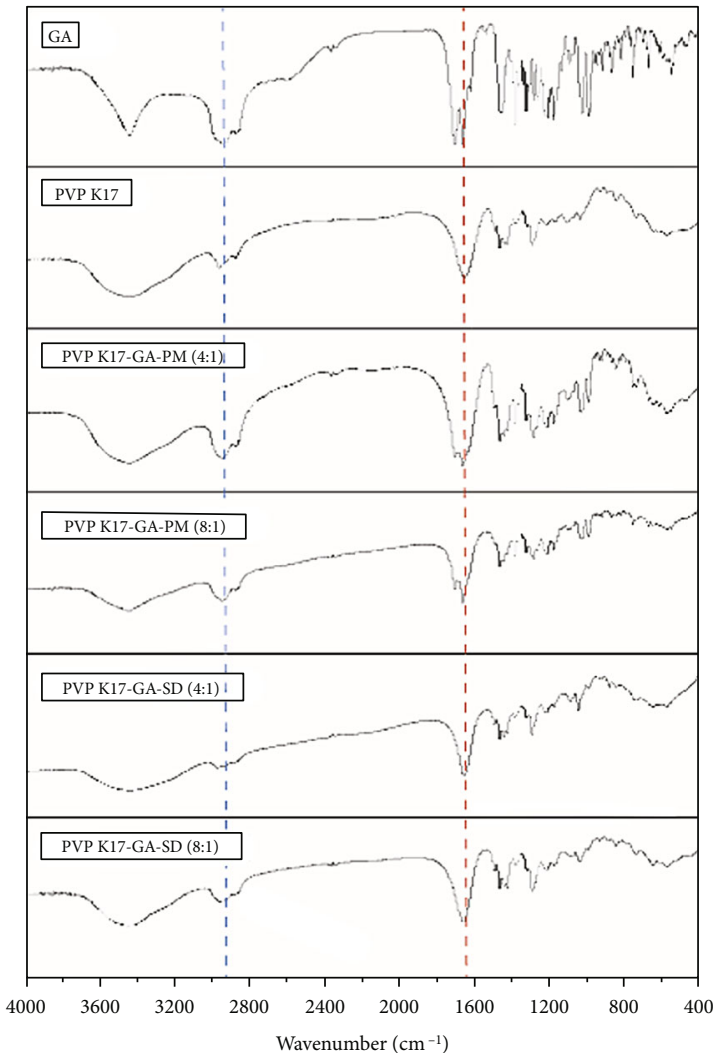

(b)

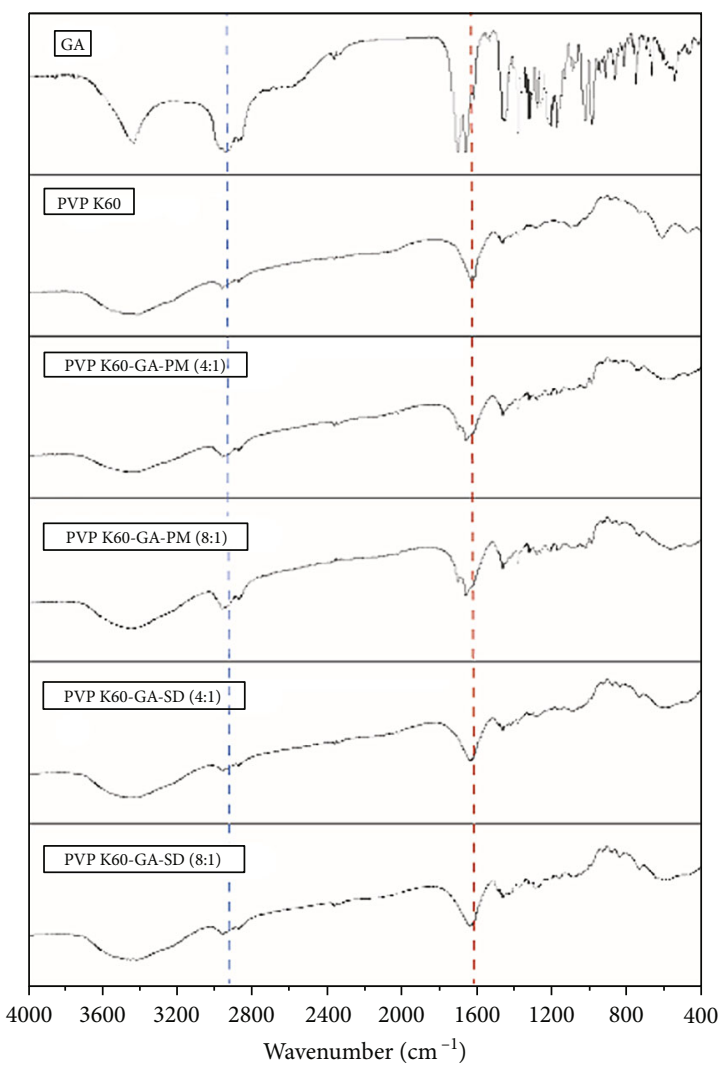

(d)

Figure 5: FTIR spectra of SDs and PMs of GA prepared using (a) PVP K15, (b) PVP K17, (c) PVP K30, and (d) PVP K60. 
with the drug, the drug crystallization is inhibited [33-35]. In order to evaluate whether this was a crystalline change of GA in SDs with different PVP molecular weight, XRD analysis was performed for the detection. As shown in Figures 4(a) and 4(d), GA exhibited multiple distinct and sharp diffraction peaks of crystal form between $3^{\circ}$ to $60^{\circ}$, indicating that GA existed in the highly crystalline form.

Four PVPs with different molecular weights showed no characteristic diffraction peaks at the range of the scanning, which indicated that they all existed in amorphous form. The crystal diffraction peaks of GA were observed in the corresponding positions of all the PMs prepared by four PVPs at different molecular weights, which suggested that the drug existed in crystalline form in all PMs.

Among the XRD spectrum of SDs, the characteristic diffraction peaks of GA of SDs prepared by PVP K30 and PVP K60 basically disappeared, indicating that GA existed in amorphous form. The characteristic diffraction peaks of GA of SDs prepared with PVP K15 and PVP K17 still can be clearly seen, indicating that GA existed in crystalline form. Therefore, it can be speculated that GA crystallization of SDs prepared with PVP K30 and PVP K60 could be significantly inhibited. This result has a positive correlation with the higher solubility of PVP K30-SDs and PVP K60-SDs compared to the other two solid dispersions.

According to the drug release model of SDs with watersoluble polymer as carrier proposed by Craig, the release of the drug from the SDs is not only related to polymer but also to drug [36]. For the PVP-GA-SDs prepared in this study, we speculate that when PVP K15, PVP K17, and PVP K30 are used as carriers, GA is mainly released as solid particles, and the dissolution rate is mainly affected by the size and crystallinity of the drug. It can be seen from the XRD spectrum that the crystallinity of PVP K30-GA-SDs is significantly lower than that of PVP K15-GA-SDs and PVP K17GA-SDs, which leads to higher chemical potential and supersaturation of the drugs in PVP K30-GA-SDs, and thus has a faster dissolution rate. On the other hand, according to the drug release model, the drug molecules must first pass through the polymer-rich diffusion layer and then be released into the bulk phase. According to the Stokes-Einstein equation, the diffusion coefficient is inversely proportional to the viscosity. Although the XRD spectrum shows that the GA in PVP K30-GA-SDs and PVP K30-GA-SDs are both transformed to amorphous, the viscosity of PVP K60 is greater than PVP K30, so when the drug is initially released from PVP K60, it is blocked by the polymer diffusion layer, so the dissolution rate of PVP K60-GA-SDs is slower than that of PVP K30-GA-SDs.

3.5. Infrared (IR) Spectrum Analysis. Many studies have shown that solid dispersions can increase drug solubility related to drug-carrier interactions, especially hydrogen bonding [37]. In this study, FTIR was used to detect potential intermolecular interactions effects of solid dispersion systems.

As seen in Figures 5(a) and 5(d), in the IR spectra of GA, the peaks of $\mathrm{GA}$ at $1,704 \mathrm{~cm}^{-1}$ and $1,664 \mathrm{~cm}^{-1}$ are attributed to $\mathrm{C}=\mathrm{O}$ in carboxylic acid and carbonyl moieties [38]. The absorption peak of phenol-OH in $\mathrm{C} 3$ was at $3,440 \mathrm{~cm}^{-1}$, and

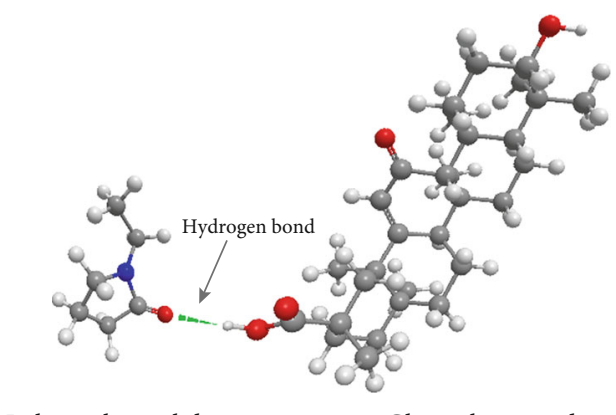

Polyvinylpyrrolidone Glycyrrhetic acid

FIgURE 6: The schematic diagram of the intermolecular hydrogen bond between GA and PVP.

absorption peak at $2,940 \mathrm{~cm}^{-1}$ is due to the stretching vibration of hydroxyl in the carboxyl group (blue dotted line). The absorption peaks (red dotted line) of $\mathrm{C}=\mathrm{O}$ were found at approximately $1,662 \mathrm{~cm}^{-1}$ in four PVPs with different molecular weights $[30,39]$.

In the IR spectra of various PMs except PVP K15-GAPMs, the two absorption peaks at $1,704 \mathrm{~cm}^{-1}$ and $1,664 \mathrm{~cm}^{-1}$ of GA can be still observed, and they overlap with the carbonyl absorption peak at $1662 \mathrm{~cm}^{-1}$ of PVPs, whereas these two absorption peaks in PVP K15-GA-PMs IR spectra tended to form a single peak. In addition, the absorption peak at $2,940 \mathrm{~cm}^{-1}$ can be clearly seen in the physical mixtures of the four molecular weight PVP and drug. However, in the IR spectra of all four SDs, $2,940 \mathrm{~cm}^{-1}$ peaks are clearly weakened, especially for PVP K30-GA-SDs and PVP K60-GASDs. Furthermore, compared with the PMs, the two peaks $\left(1,704 \mathrm{~cm}^{-1}\right.$ and $\left.1,664 \mathrm{~cm}^{-1}\right)$ of GA turn into a single peak, and the peak intensity is weakened and blunted with different degrees. This can be seen more clearly in IR spectra of PVP K30-GA-SDs and PVP K60-SDs. These results suggest that the hydroxyl in the carboxyl group in GA may form a hydrogen bond with $\mathrm{C}=\mathrm{O}$ of PVPs, which changes the characteristics of IR spectra; the schematic diagram of the intermolecular hydrogen bond between GA and PVP is shown in Figure 6 . This result is also consistent with that detected by XRD. The hydrogen bonding effect of PVP K30-GA-SDs and PVP K60-GA-SDs is more obvious, and their XRD characteristic peak strength is also weaker, which indicates that the hydrogen bonding interaction between GA and PVPs leads to the formation of amorphous structure of glycyrrhetic acid. In addition, because the hydroxyl absorption peak of GA at $3440 \mathrm{~cm}^{-1}$ overlaps with the characteristic absorption peak of PVP at $3710-3020 \mathrm{~cm}^{-1}$, we cannot observe whether the C3 hydroxyl absorption peak of GA in GA-SDs has obvious migration or passivation. Therefore, through infrared spectroscopy, we cannot infer whether the C3 hydroxyl group has formed a hydrogen bond with the carbonyl group of PVP.

3.6. Raman Spectroscopy. Raman spectroscopy and infrared spectroscopy are both vibrational spectra of molecules, and they are complementary. Generally speaking, the asymmetric vibrations of polar groups are more suitable for detection by infrared. The vibration of nonpolar groups and the molecular 


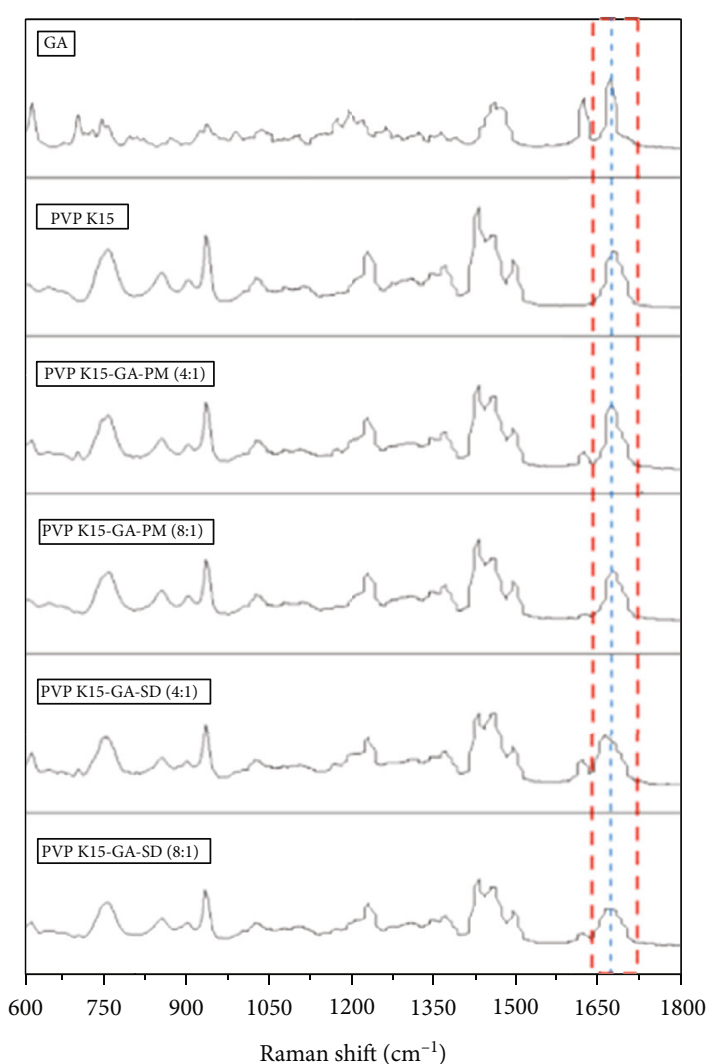

(a)

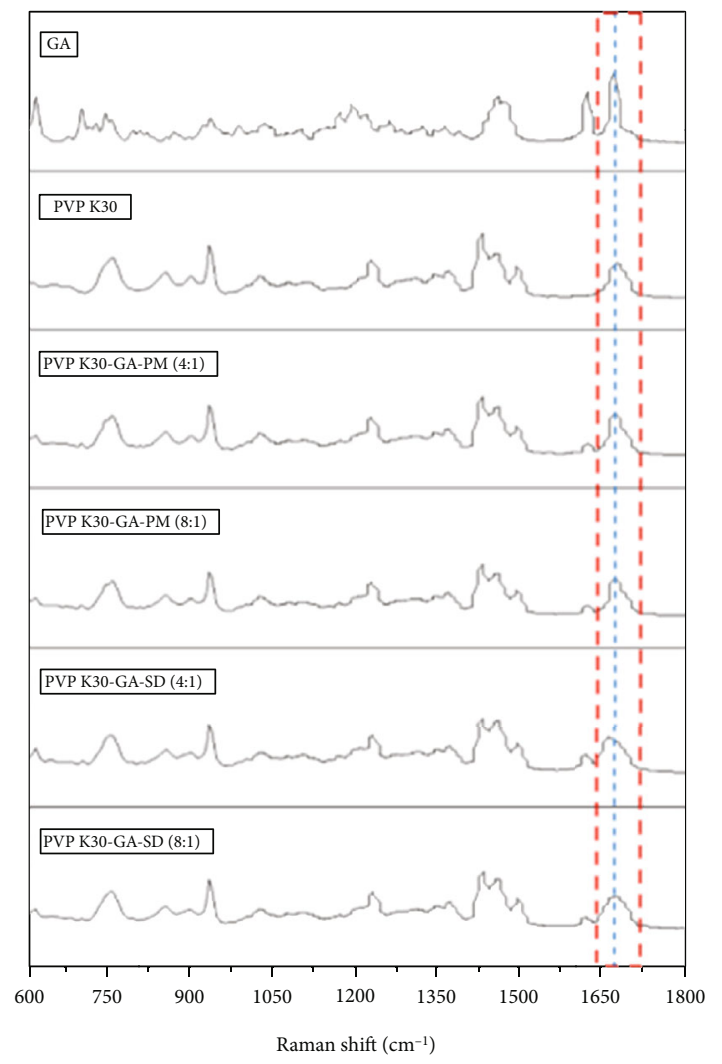

(c)

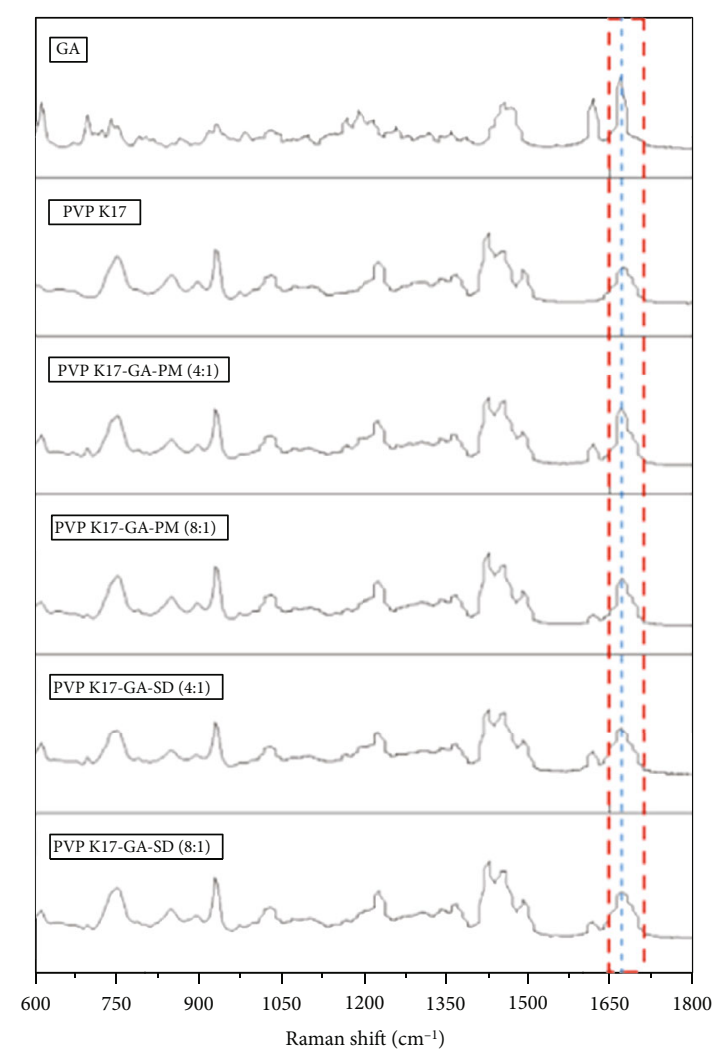

(b)

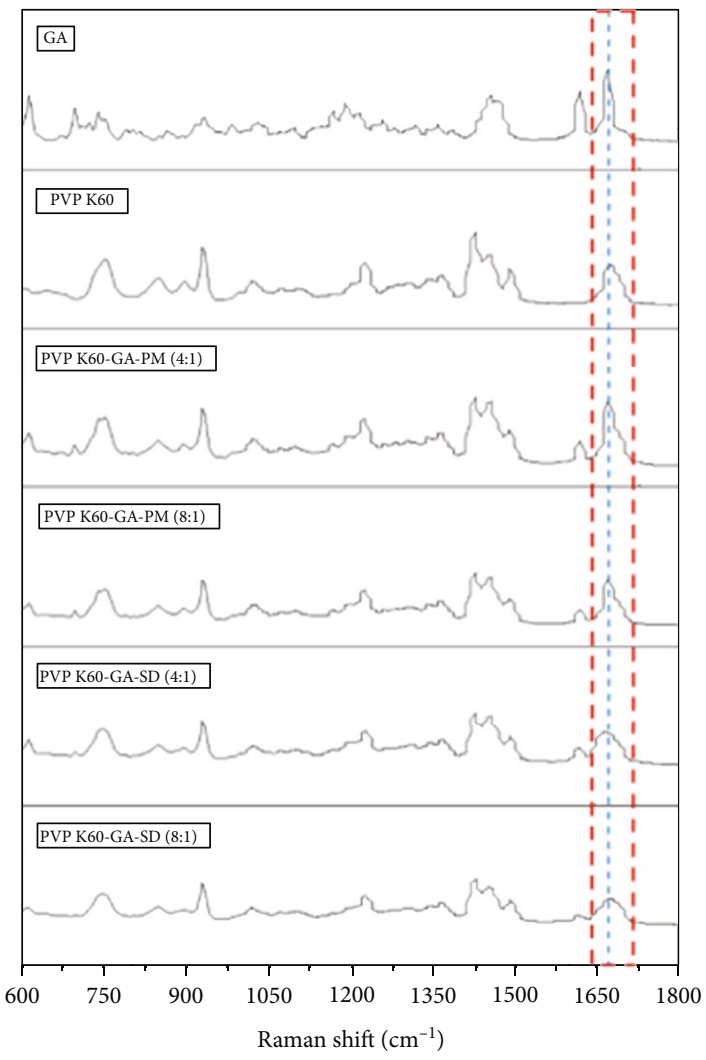

(d)

Figure 7: Raman spectra of SDs and PMs of GA prepared using (a) PVP K15, (b) PVP K17, (c) PVP K30, and (d) PVP K60. 


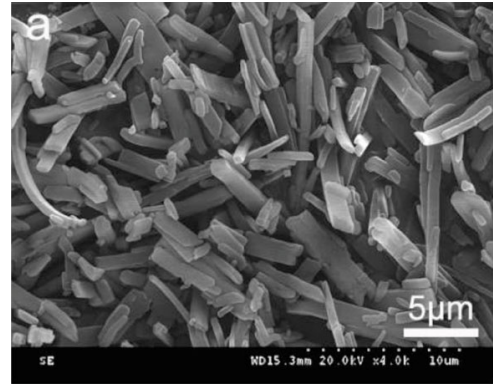

(a)

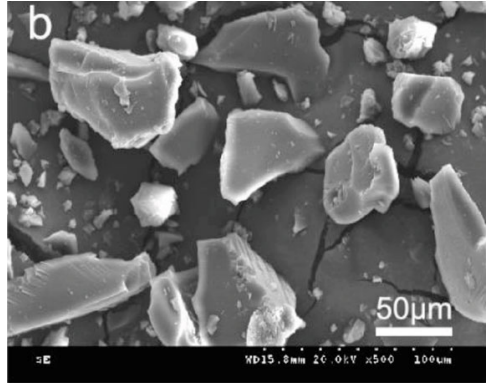

(b)

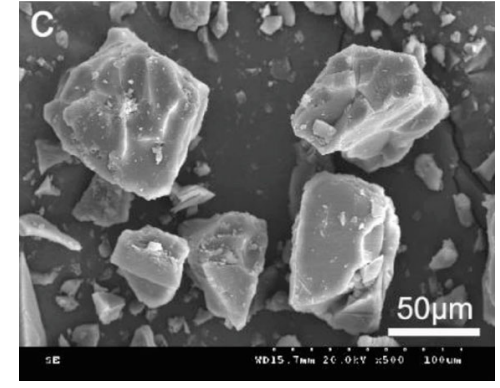

(c)

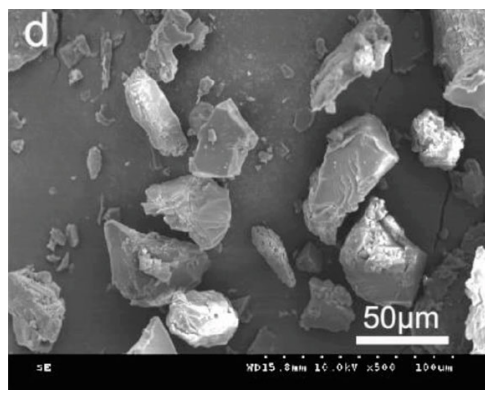

(d)

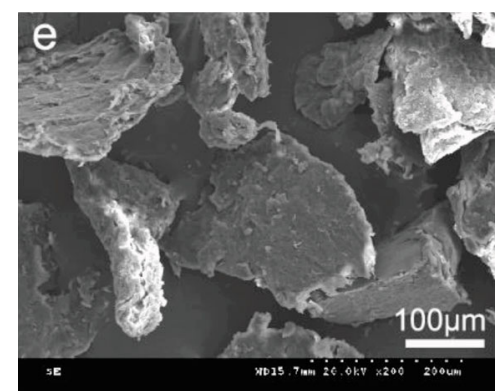

(e)

FIgURe 8: SEM images of (a) pure GA and SDs prepared using (b) PVP K15, (c) PVP K17, (d) PVP K30, and (e) PVP K60.

vibration of full symmetry are more suitable for detection by Raman scattering. Some studies have used Raman spectroscopy to study the interaction of polymers and drugs in solid dispersions [40, 41]. Therefore, in order to compare the effects of different molecular weight carriers on the structure of solid dispersions, a Raman spectrum analysis was performed on each sample.

As presented in Figures 7(a) and 7(d), pure GA had two sharp characteristic peaks at $1620 \mathrm{~cm}^{-1}$ and $1,670 \mathrm{~cm}^{-1}$, which is attributed to stretching vibrations of the carbonyl moiety and -COO- group [11]. Four different molecular weight PVPs also showed a significant scattering peak of carbonyls at $1670 \mathrm{~cm}^{-1}$ [42], and the peak shape is not sharp. However, a sharp peak-shaped scattering peak (red rectangle frame) can be observed at $1670 \mathrm{~cm}^{-1}$ in PMs (blue dotted line) of four different molecular weight PVPs and GA, which indicated that the PM scattering peak at $1670 \mathrm{~cm}^{-1}$ is the result of the overlapping of two scattering peaks without chemical reaction. Compared with the physical mixtures, the scattering peaks of GA-SDs prepared using four molecular weight PVPs at $1670 \mathrm{~cm}^{-1}$ are obviously broader and weaker (red rectangle frame). This is consistent with XRD results. Moreover, in the spectra of PVP-GA-SDs (4: 1) prepared from four molecular weight PVPs, the peak at $1670 \mathrm{~cm}^{-1}$ all move towards the low wavenumber region (light blue dotted line). However, in the PVP-GA-SDs (8:1) spectrum, the scattering peak at $1670 \mathrm{~cm}^{-1}$ has not shifted. This may be due to the low content of GA in the sample, which caused the hydrogen bonding in the surface area to be dispersed and weakened and did not cause scattering peak change. From this result, it can be speculated that the carbonyl group of PVP forms a hydrogen bond with the hydroxyl group of glycyrrhetic acid molecule.
3.7. Scanning Electron Microscope (SEM) Analysis. Figure $8(\mathrm{a})$ is the SEM image of GA, showing that GA is rod-like and its crystal shape is clear. Figures $8(\mathrm{~b})$ and $8(\mathrm{e})$ are SEM images of the PVP-GA-SDs $(8: 1)$, presenting the SDs as irregular masses with the rod-like crystal GA unobservable. This suggests that glycyrrhetic acid has been uniformly dispersed in PVP in amorphous or microcrystalline form. Compared with PVP K60-GA-SDs, the surface of the solid dispersions prepared with PVP K15, PVPK17, and PVP K30 is smoother. This may be due to the larger molecular weight of PVP K60, resulting in greater cohesion. In addition, the average particle size of SDs prepared with PVP K60 as a carrier was larger than the sizes of samples with the other three molecular weight PVPs. However, the solubility and dissolution studies have shown that the performance of PVP K60-SDs was better than PVP K15-SDs and PVP K17SDs, which indicates that the particle size of the solid dispersion has no significant effect on dissolution. According to the Noyes-Whitney equation, the dissolution rate of a drug is related to the particle size of the drug [22]. Although the particle size of PVP K60-SDs was larger than samples prepared using PVP K15 and PVP K17, the dispersion efficacy of GA in the PVP K60 may be better, which leads to better solubility and dissolution performance.

\section{Conclusions}

The results confirmed that the molecular weight of a carrier has a significant influence on the drug dissolution rate from solid dispersion systems. For specific drugs, the effect of carrier molecular weight on its dissolution properties is unique. According to our research, when PVP is used as the carrier and the solvent method is used to prepare the glycyrrhetic 
acid solid dispersions, the effect of PVP molecular weight on the solubility and dissolution of the drug is not monotonic. Among all PVPs, the dissolution effect of glycyrrhetic acid solid dispersion was best when PVP K30 was used as the carrier. And the effect of carrier-drug weight ratio on the dissolution effect is also obvious. The dissolution rate of the carrier-drug weight ratio of $8: 1$ is faster than that of the 4:1 sample. This can be useful for the choice of the proper materials for GA applications. It is expected that the PVP K30-GA-SDs can be used as an ideal material for the development of glycyrrhetic acid oral products. On the other hand, in this study, we only used the solvent method and only investigated the effects of four molecular weight carriers (including PVP K15, PVP K17, PVP K30, and PVP K60) on the properties of solid dispersions. Perhaps other preparation methods (such as electrospinning and hot melt extrusion) or PVP with other molecular weights would have a better dissolution rate, which requires further research in the follow-up.

\section{Data Availability}

The data used to support the findings of this study are included within the article.

\section{Conflicts of Interest}

The authors declare no conflicts of interest.

\section{Acknowledgments}

This research was funded by the University Nursing Program for Young Scholars with Creative Talents in Heilongjiang Province, China (UNPYSCT-2017168) and the Foundation of Educational Committee in Heilongjiang Province, China (2016-KYYWF-0856).

\section{References}

[1] Y. Cai, Y. Xu, H. F. Chan, X. Fang, C. He, and M. Chen, “Glycyrrhetinic acid mediated drug delivery carriers for hepatocellular carcinoma therapy," Molecular Pharmaceutics, vol. 13, no. 3, pp. 699-709, 2016.

[2] T. Akao, T. Hayashi, K. Kobashi et al., "Intestinal bacterial hydrolysis is indispensable to absorption of $18 \beta$-glycyrrhetic acid after oral administration of glycyrrhizin in rats," Journal of Pharmacy and Pharmacology, vol. 46, no. 2, pp. 135-137, 1994.

[3] A. M. Aly, L. Al-Alousi, and H. A. Salem, "Licorice: a possible anti-inflammatory and anti-ulcer drug," AAPS PharmSciTech, vol. 6, no. 1, pp. E74-E82, 2005.

[4] M. E. Hardy, "18 $\beta$-glycyrrhetinic acid inhibits rotavirus replication in culture," Virology Journal, vol. 9, no. 1, p. 96, 2012.

[5] A. Roohbakhsh, M. Iranshahy, and M. Iranshahi, "Glycyrrhetinic acid and its derivatives: anti-cancer and cancer chemopreventive properties, mechanisms of action and structurecytotoxic activity relationship," Current Medicinal Chemistry, vol. 23, no. 5, pp. 498-517, 2016.

[6] P. Kuang, W. Zhao, W. Su et al., "18 $\beta$-glycyrrhetinic acid inhibits hepatocellular carcinoma development by reversing hepatic stellate cell-mediated immunosuppression in mice," International Journal of Cancer, vol. 132, no. 8, pp. 18311841, 2013.

[7] Y. Lei, Y. Kong, H. Sui, J. Feng, R. Zhu, and W. Wang, "Enhanced oral bioavailability of glycyrrhetinic acid via nanocrystal formulation," Drug Delivery and Translational Research, vol. 6, no. 5, pp. 519-525, 2016.

[8] X. Zhao, Y. Fan, D. Wang et al., "Immunological adjuvant efficacy of glycyrrhetinic acid liposome against Newcastle disease vaccine," Vaccine, vol. 29, no. 52, pp. 9611-9617, 2011.

[9] C. Puglia, L. Rizza, M. Drechsler, and F. Bonina, "Nanoemulsions as vehicles for topical administration of glycyrrhetic acid: characterization and in vitro and in vivo evaluation," Drug Delivery, vol. 17, no. 3, pp. 123-129, 2010.

[10] H. Onishi, H. Takahashi, and Y. Machida, "Preparation and evaluation of glycyrrhetic acid-containing microparticles as an anti-hepatotoxic system," Drug Development Research, vol. 66, no. 3, pp. 189-199, 2005.

[11] L. Dong, Y. Mai, Q. Liu, W. Zhang, and J. Yang, "Mechanism and improved dissolution of glycyrrhetinic acid solid dispersion by alkalizers," Pharmaceutics, vol. 12, no. 1, p. 82, 2020.

[12] U. H. Gala, D. A. Miller, and R. O. Williams, "Harnessing the therapeutic potential of anticancer drugs through amorphous solid dispersions," Biochimica et Biophysica Acta (BBA) Reviews on Cancer, vol. 1873, no. 1, article 188319, 2020.

[13] C. L. N. Vo, C. Park, and B. J. Lee, "Current trends and future perspectives of solid dispersions containing poorly watersoluble drugs," European Journal of Pharmaceutics and Biopharmaceutics, vol. 85, no. 3, pp. 799-813, 2013.

[14] F. Hallouard, L. Mehenni, M. Lahiani-Skiba, Y. Anouar, and M. Skiba, "Solid dispersions for oral administration: an overview of the methods for their preparation," Current Pharmaceutical Design, vol. 22, no. 32, pp. 4942-4958, 2016.

[15] O. A. Sammour, M. A. Hammad, N. A. Megrab, and A. S. Zidan, "Formulation and optimization of mouth dissolve tablets containing rofecoxib solid dispersion," AAPS PharmSciTech, vol. 7, no. 2, pp. e167-e175, 2006.

[16] G. A. Digenis, D. A. Wells, J. M. Ansell, and L. Blecher, "Disposition of $[14 \mathrm{C}]$ povidone after oral administration to the rat," Food and Chemical Toxicology, vol. 25, no. 3, pp. 241243, 1987.

[17] T. D. Silva, V. T. Arantes, J. A. L. C. Resende, N. L. Speziali, R. B. De Oliveira, and C. D. Vianna-Soares, "Preparation and characterization of solid dispersion of simvastatin," Drug Development and Industrial Pharmacy, vol. 36, no. 11, pp. 1348-1355, 2010.

[18] V. Tantishaiyakul, N. Kaewnopparat, and S. Ingkatawornwong, "Properties of solid dispersions of piroxicam in polyvinylpyrrolidone," International Journal of Pharmaceutics, vol. 181, no. 2, pp. 143-151, 1999.

[19] L. D. S. Alves, M. F. de La Roca Soares, C. T. de Albuquerque et al., "Solid dispersion of efavirenz in PVP K-30 by conventional solvent and kneading methods," Carbohydrate Polymers, vol. 104, no. 1, pp. 166-174, 2014.

[20] P. Kanaujia, P. Poovizhi, W. K. Ng, and R. B. H. Tan, “Amorphous formulations for dissolution and bioavailability enhancement of poorly soluble APIs," Powder Technology, vol. 285, pp. 2-15, 2015.

[21] D. D. Bavishi and C. H. Borkhataria, "Spring and parachute: how cocrystals enhance solubility," Progress in Crystal Growth and Characterization of Materials, vol. 62, no. 3, pp. 1-8, 2016. 
[22] C. Leuner and J. Dressman, "Improving drug solubility for oral delivery using solid dispersions," European Journal of Pharmaceutics and Biopharmaceutics, vol. 50, no. 1, pp. 47-60, 2000.

[23] M. M. Knopp, J. H. Nguyen, C. Becker et al., "Influence of polymer molecular weight on in vitro dissolution behavior and in vivo performance of celecoxib:PVP amorphous solid dispersions," European Journal of Pharmaceutics and Biopharmaceutics, vol. 101, pp. 145-151, 2016.

[24] N. M. Najib, M. Suleiman, and A. Malakh, "Characteristics of the in vitro release of ibuprofen from polyvinylpyrrolidone solid dispersions," International Journal of Pharmaceutics, vol. 32, no. 2-3, pp. 229-236, 1986.

[25] S. Ugaonkar, A. C. Nunes, and T. E. Needham, "Effect of nscCO2 on crystalline to amorphous conversion of carbamazepine," International Journal of Pharmaceutics, vol. 333, no. 12, pp. 152-161, 2007.

[26] B. Yang, L. Wu, J. Ke et al., "Effects of polymer/surfactant as carriers on the solubility and dissolution of fenofibrate solid dispersion," AAPS PharmSciTech, vol. 20, no. 3, p. 102, 2019.

[27] N. Yagi, Y. Terashima, H. Kenmotsu, H. Sekikawa, and M. Takada, "Dissolution behavior of probucol from solid dispersion systems of probucol-polyvinylpyrrolidone," Chemical and Pharmaceutical Bulletin, vol. 44, no. 1, pp. 241-244, 1996.

[28] J. S. Choi and J.-S. Park, "Design of PVP/VA S-630 based tadalafil solid dispersion to enhance the dissolution rate," European Journal of Pharmaceutical Sciences, vol. 97, pp. 269276, 2017.

[29] A. Paradkar, A. A. Ambike, B. K. Jadhav, and K. R. Mahadik, "Characterization of curcumin-PVP solid dispersion obtained by spray drying," International Journal of Pharmaceutics, vol. 27, no. 1-2, pp. 281-286, 2004.

[30] F. Frizon, J. de Oliveira Eloy, C. M. Donaduzzi, M. L. Mitsui, and J. M. Marchetti, "Dissolution rate enhancement of loratadine in polyvinylpyrrolidone K-30 solid dispersions by solvent methods," Powder Technology, vol. 235, pp. 532-539, 2013.

[31] N. Fukuda, N. Higuchi, M. Ohno, H. Kenmochi, H. Sekikawa, and M. Takada, "Dissolution behavior of prednisolone from solid dispersion systems with cyclodextrins and polyvinylpyrrolidone," Chemical and Pharmaceutical Bulletin, vol. 34, no. 3, pp. 1366-1369, 1986.

[32] M. Barzegar-Jalali, H. Valizadeh, M.-R. S. Shadbad et al., "Cogrinding as an approach to enhance dissolution rate of a poorly water-soluble drug (gliclazide)," Powder Technology, vol. 197, no. 3, pp. 150-158, 2010.

[33] P. Gupta, V. K. Kakumanu, and A. K. Bansal, "Stability and solubility of celecoxib-PVP amorphous dispersions: a molecular perspective," Pharmaceutical Research, vol. 21, no. 10, pp. 1762-1769, 2004.

[34] L. S. Taylor and G. Zografi, "Spectroscopic characterization of interactions between PVP and indomethacin in amorphous molecular dispersions," Pharmaceutical Research, vol. 14, no. 12, pp. 1691-1698, 1997.

[35] G. Van den Mooter, M. Wuyts, N. Blaton et al., "Physical stabilisation of amorphous ketoconazole in solid dispersions with polyvinylpyrrolidone K25," European Journal of Pharmaceutical Sciences, vol. 12, no. 3, pp. 261-269, 2001.

[36] D. Q. M. Craig, "The mechanisms of drug release from solid dispersions in water-soluble polymers," International Journal of Pharmaceutics, vol. 231, no. 2, pp. 131-144, 2002.

[37] S. Sinha, M. Ali, S. Baboota, A. Ahuja, A. Kumar, and J. Ali, "Solid dispersion as an approach for bioavailability enhance- ment of poorly water-soluble drug ritonavir," AAPS PharmSciTech, vol. 11, no. 2, pp. 518-527, 2010.

[38] Y. Zheng, Y. Wu, W. Yang, C. Wang, S. Fu, and X. Shen, "Preparation, characterization, and drug release in vitro of chitosan-glycyrrhetic acid nanoparticles," Journal of Pharmaceutical Sciences, vol. 95, no. 1, pp. 181-191, 2006.

[39] S. Sethia and E. Squillante, "Solid dispersion of carbamazepine in PVP K30 by conventional solvent evaporation and supercritical methods," International Journal of Pharmaceutics, vol. 272, no. 1-2, pp. 1-10, 2004.

[40] N. Furuyama, S. Hasegawa, T. Hamaura et al., "Evaluation of solid dispersions on a molecular level by the Raman mapping technique," International Journal of Pharmaceutics, vol. 361, no. 1-2, pp. 12-18, 2008.

[41] S. Keratichewanun, Y. Yoshihashi, N. Sutanthavibul, K. Terada, and J. Chatchawalsaisin, "An investigation of nifedipine miscibility in solid dispersions using raman spectroscopy," Pharmaceutical Research, vol. 32, no. 7, pp. 24582473, 2015.

[42] G. P. Andrews, O. A. Abudiak, and D. S. Jones, "Physicochemical characterization of hot melt extruded bicalutamidepolyvinylpyrrolidone solid dispersions," Journal of Pharmaceutical Sciences, vol. 99, no. 3, pp. 1322-1335, 2010. 